\title{
呼吸训练联合核心稳定性训练治疗 儿童痉孪型脑性㿈㾔疗效研究
}

\author{
胡碧浓 ${ }^{1}$, 张 凯 ${ }^{1}$, 被 芳 ${ }^{1}$, 王春华 ${ }^{2}$, 周文䓉 ${ }^{2}$, 艾 坤 ${ }^{1^{*}}$, 张 泓 ${ }^{1^{*}}$ \\ 1 湖南中医药大学针茨推拿学院, 湖南 长沙 410208 ; \\ 2 湘雅博爱康复医院, 湖南长沙 410000 \\ * 通信作者: 张泓 ,E-mail :zh5381271@sina.com; 艾坤,E-mail:55509095@qq.com
}

收稿日期: 2020-08-02; 接受日期 : 2020-09-27

基金项目: 湖南省大学生研究性学习和创新性实验计划项目 (2018-434); 湖南省残疾人康复科研项目 (2019XK014)

DOI: $10.3724 /$ SP.J.1329.2020.06006

开放科学(资源服务) 标识码 (OSID)

摘要 目的: 观察呼吸训练联合核心稳定性训练对痉孪型脑性痽痿 (SCP) 儿童运动功能、平衡功能及日常生 活活动能力的影响。方法: 选择 2019 年 3 月-12 月在湘雅博爱康复医院儿童康复科治疗的 SCP 儿童 74 例, 采用随机数字表法分为对照组和观察组, 每组 37 例。治疗过程中, 每组脱落 2 例, 最后每组纳入 35 例。对照 组在常规康复训练基础上, 给予核心稳定性训练。常规康复训练主要包括牵伸训练、关节活动度训练、作业治 疗, $10 \mathrm{~min} /$ 次。核心稳定性训绕包括:搭桥训练、仰卧起坐训练、悬吊系统训练、Bobath 球训练, $40 \mathrm{~min} /$ 次。观 察组在对照组常规康复训练基础上,将核心稳定性训练时间由 $40 \mathrm{~min} /$ 次缩减为 $20 \mathrm{~min} /$ 次, 然后增加呼吸 训练, $20 \mathrm{~min} /$ 次。呼吸训练包括: 腹式呼吸训练 (7 min/次)、扩胸运动训练 (3 min/次)、呼吸借助法训练 (4 min/次)、抗阻呼气训䋆 $(6 \mathrm{~min} /$ 次)。以上项目均训练 2 次/ $\mathrm{d}, 5$ 次/周, 持续训练 3 个月。在治疗前及治疗 3 个月后, 分别采用粗大运动功能测试量表(GMFM-88) 中 D、E 功能区评估儿童站立、行走、跑和跳的粗大 运动功能; 采用 Berg 平衡量表(BBS)评估平衡功能; 采用 Barthel 指数(BI)评估日常生活活动能力。结果: 治 疗前,2 组 GMFM-88 的 D 和 E 功能区评分、BBS 评分和 BI 评分比较无明显区别, 差异均无统计学意义 $(P>0.05)$ 。与治疗前比较, 2 组治疗 3 个月后 GMFM-88 的 D 和 $\mathrm{E}$ 功能区评分、BBS 评分、BI 评分均明显提 高, 差异有统计学意义 $(P<0.05)$; 与对照组比较, 观察组治疗 3 个月后 GMFM-88 D 和 E 功能区评分、BBS 评分、 $\mathrm{BI}$ 评分均明显更高, 差异有统计学意义 $(P<0.05)$ 。结论: 呼吸训练联合核心稳定性训练可有效提高 $\mathrm{SCP}$ 儿童粗大运动功能、平衡能力和日常生活活动能力,值得临床推广。

关键词痉孪型脑性㿈疾; 呼吸训练;核心稳定性训练;运动功能;平衡能力;日常生活活动能力

脑性痽瘾是一组持续存在的中枢性运动和姿 势发育障碍、活动受限症候群,主要是由于发育中 胎儿或肾幼儿脑部非进行性损伤所致 ${ }^{[1]}$ 。痉挛型脑 性痽瘾 (spastic cerebral palsy, SCP) 是脑性痽瘑最常 见的一种类型,约占脑性㿈疾总数的 $60 \% \sim 70 \%$ 。 由于中枢神经系统的损伤,主要表现为运动和姿势 发育异常, 肌张力增高, 核心稳定性下降,逐渐形成 异常的运动模式,骨骼发育畸形, 肌肉孪缩, 儿童运 动发育受阻 ${ }^{[3]}$ 。平衡障碍是 SCP 儿童常见的功能障
碍, 这对儿童的行走、跑跳以及日常生活能力产生 较大影响。此外, SCP 儿童因胸廓畸形、呼吸肌无力 和呼吸模式异常等原因 ${ }^{[4]}$, 导致呼吸无法正常进行; 膈肌、腹横肌等核心肌群 (重要的呼吸肌) 肌力下 降,脑症儿童核心稳定性出现不同程度的减退 ${ }^{[5]}$, 从 而延缓了康复进程, 给家庭和社会带来较为沉重的 负担 ${ }^{[6]}$ 。因此, 寻求有效的康复方法, 预防 SCP 几童 身体早期发育的异常, 提高儿童的运动功能、平衡 能力和日常生活能力, 显得尤为重要。有研究显示,

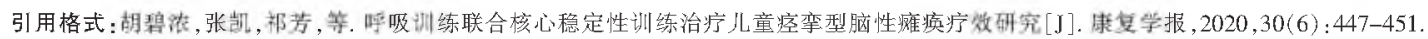

HU B N, ZHANG K, OI F, et al. Effect of respiratory training combined with core stability training for children with spastic cerebral palsy [J]. Rehabilitation Medicine, $2020,30(6): 447-451$.

DOI: $10.3724 /$ SP.J.1329.2020.06006 
常规训练联合核心稳定性训练可以提高 SCP 儿童 的粗大运动功能、平衡能力和日常生活能力 ${ }^{[7-8]}$; 呼 吸肌训练有助于提高脑性㿈疾儿童核心区域的稳 定和运动功能的发育 ${ }^{[9]}$ 。本研究在常规训练基础上, 应用呼吸训练配合核心稳定性训练治疗 SCP 儿童, 取得良好疗效。现报道如下。

\section{1 临床资料}

\section{1 病例选择标准}

1.1.1 诊断标准符合《中国脑性㿈疾康复指南 (2015): 第一部分》中小儿脑症诊断及分型标准 ${ }^{[1]}$ : (1) 中枢性运动障碍持续存在; (2) 运动和姿势发育 异常; (3) 反射发育异常; (4) 肌张力及肌力异常; (5) 磁 共振成像辅助诊断。

1.1.2 纳人标准 (1) 年龄: 3 9 岁; (2) 有较好的依 从性, 能理解指令并配合训练; (3) 粗大运动功能分 级系统 (gross motor function classification system, GMFCS) ${ }^{[10]}$ 评定为 $\mathrm{I} \sim$ III 级; (4) 病情稳定; (5) 监护
人知晓研究方案并签署知情同意书。

1.1.3 排除标准 (1) 合并癫㾁或严重心肺疾病; (2) 有严重的肌肉、骨关节等器质性病变; (3) 人组前 半年曾接受选择性脊神经后根切断术等外科手术 或注射肉毒素。

1.1.4 脱落标准 (1) 治疗依从性不高, 无法完成全 程观察者; (2) 中途出院或转院者。

\section{2 一般资料}

选取 2019 年 3 月- 12 月在湘雅博爱康复医院 儿童康复科接受治疗的 SCP 儿童 74 例, 按照随机。 数字表法分为对照组和观察组, 每组 37 例。治疗过 程中, 因个人原因中途放弃治疗 4 例, 每组脱落 2 例, 分别纳人 35 例。2 组性别、年龄、临床分型和 GMFCS 分级等一般资料比较, 差异均无统计学意义 $(P>$ $0.05)$, 具有可比性。见表 1 。本研究严格遵从《世界 赫尔辛基宣言》关于临床试验的伦理相关要求, 并 经湘雅博爱康复医院伦理委员会审批通过 (审批号: 20190107)。

表 12 组一般资料比较 $(\bar{x} \pm s)$

Table 1 Comparison of general data between two groups $(\bar{x} \pm s)$

\begin{tabular}{|c|c|c|c|c|c|c|c|c|c|c|}
\hline \multirow{2}{*}{ 组 别 } & \multirow{2}{*}{$n$} & \multicolumn{2}{|c|}{ 性别 } & \multirow{2}{*}{ 年龄/岁 } & \multicolumn{3}{|c|}{ 临床分型 } & \multicolumn{3}{|c|}{ GMFCS 分级 } \\
\hline & & 男 & $\overline{\text { 女 }}$ & & 偏瘫 & 双瘫 & $\begin{array}{l}\text { 四肢㿈 } \\
\end{array}$ & I 级 & II 级 & III 级 \\
\hline 对照组 & 35 & 23 & 12 & $5.06 \pm 1.55$ & 10 & 21 & 4 & 12 & 13 & 10 \\
\hline 观察组 & 35 & 25 & 10 & $5.23 \pm 1.55$ & 12 & 20 & 3 & 13 & 11 & 11 \\
\hline
\end{tabular}

\section{2 方 法}

\section{1 治疗方法}

2.1.1 对照组 在常规康复训练基础上, 给予核心 稳定性训练 ${ }^{[11]}, 2$ 次/d,5 次/周, 持续训练 3 个月。 具体如下:

2.1.1.1 常规康复训练 (1) 牵伸训练: 对躯干和四 肢痉挛肌群进行被动牵拉, 降低肌肉肌张力; (2) 关 节活动度训练: 治疗师被动活动 SCP 儿童大关节 (如髋关节、肩关节等), 并诱导脑症儿童主动配合 关节范围练习, 增加关节活动度; (3) 作业治疗: 针对 生活中日常活动进行训练, 如穿衣服、洗漱活动等, 增强日常生活活动能力。10 min/次。

2.1.1.2 核心稳定性训练 (1) 搭桥训练: 儿童仰卧 位, 头与脊柱保持中线, 屈髋屈膝, 双脚与肩同宽, 抬高慰部成桥面形状, 骨盆保持稳定, 此动作可激 活腹直肌、腹外斜肌、腹内斜肌; (2) 仰卧起坐 : 儿童 仰卧位, 背后可垫楔形垫, 屈髋屈滕, 头及躯干用力 向前屈曲, 双手往前伸直, 此动作可激活腹横肌、腹 直肌、腹内外斜肌; (3) 悬吊系统训练 : 儿童取俯卧
位, 前臂伸直支撑, 悬吊环置于双踝处, 使儿童身体 平直保持姿势, 主要激活竖脊肌和腹直肌; 儿童仰卧 位于训练垫上, 踝关节放在悬吊环上, 抬高臂部, 保 持姿势, 可激活多裂肌; 儿童站立位, 双脚踩在悬吊 带上, 双手握住悬吊绳, 保持身体直立稳定, 让儿童 感知深层稳定肌群收缩; 儿童坐于悬吊船内, 在船 晃动时, 努力保持坐位平衡, 悬吊船静止时, 左右旋 转躯干, 此动作激活腹内外斜肌; (4) Bobath 球训练: 儿童坐于球上, 治疗师双手扶住儿童髋部, 使球前 后左右滚动, 儿童努力调整身体, 保持坐位正中位, 可激活竖脊肌、髂腰肌及腰方肌。40 min/次。

2.1.2 观察组 观察组在对照组常规康复训练的 基础上,将核心稳定性训练的时间由 $40 \mathrm{~min} /$ 次缩短 为 $20 \mathrm{~min} /$ 次, 然后给予呼吸训练, $20 \mathrm{~min} /$ 次, 2 次/ $\mathrm{d}$, 5 次/周, 持续训练 3 个月。呼吸训练方法 ${ }^{[12]}$ 如下:

2.1.2.1 腹式呼吸训练儿童取坐位, 治疗师位于 儿童身后, 一手置于儿童腹部, 儿童经鼻缓慢深吸气 时最大程度隆起腹部, 下胸廓胁骨稍微往外扩张, 然 后由口缓慢呼气, 呼气末儿童腹部塌陷, 肋骨回收, 可促进膈肌上移, 提高膈肌活动度, 腹式呼吸训练每 
组重复 10 次,每组训练后休息 $10 \sim 15 \mathrm{~s} 。 7 \mathrm{~min} /$ 次。 2.1.2.2 扩胸运动训练 儿童取坐位, 治疗师坐于儿 童身后, 协助儿童在吸气时进行双上肢屈曲、外展 和外旋训练, 慢慢呼气时进行双上肢伸展、内收和 内旋训练。 $3 \mathrm{~min} /$ 次。

2.1.2.3 呼吸训练借助法儿童取仰卧位, 治疗师 位于儿童身体一侧, 把握儿童呼吸节奏, 在儿童呼 气时进行徒手压迫胸部并缓慢增加压迫强度, 吸 气时胸廓扩张范围增加, 逐渐延长每次呼吸时间。 $4 \mathrm{~min} /$ 次。

2.1.2.4 抗阻呼气训练儿童取舒适体位为坐位, 用趣味性气笛进行呼气训练, 让儿童先深吸气, 然 后尽最大努力呼出气体, 延长呼气时间。每组训练 10 次, 视儿童呼吸肌力评估情况, 适当调整训练次 数。6 $\mathrm{min} /$ 次。

\section{2 观察指标}

于治疗前和治疗 3 个月后分别采用以下指标进 行评定:

2.2.1 运动功能评定 采用粗大运动功能量表 (gross motor function measure scale-88, GMFM -88) 进行粗大运动功能的评估 ${ }^{[13]}$ 。本研究选用 GMFM-88
量表的 D、E 功能区进行评分。 D 功能区有 13 项, 每 项 $0 \sim 3$ 分, 总分 39 分。 $\mathrm{E}$ 功能区有 24 项, 每项 $0 \sim$ 3 分, 总分 72 分。评分越高, 代表粗大运动功能越好。 2.2.2 平衡功能评定 采用 Berg 平衡量表(Berg balance scale, BBS) 进行平衡功能评定 ${ }^{[14]}$, 共包括 14 个项目, 每项 $0 \sim 4$ 分, 总分 56 分, 评分越高, 代表 平衡能力越好。

2.2.3 日常生活能力评定 采用 Barthel 指数 (Barthel index, BI) 进行日常生活活动能力评定 ${ }^{[15]}$, 共 10 个项 目, 每项 $0 \sim 10$ 分, 总分 100 分, 评分越高, 代表日常 生活活动能力越好。

\section{3 统计学方法}

采用 SPSS 22.0 统计软件进行数据分析。计量资 料符合正态分布采用 $(\bar{x} \pm s)$ 表示, 组间比较采用两独 立样本 $t$ 检验, 组内比较采用两配对样本 $t$ 检验; 计 数资料采用 $\chi^{2}$ 检验。 $P<0.05$ 为差异有统计学意义。

\section{3 结 果}

\subsection{2 组治疗前后 GMFM-88 评分比较} 见表 2 。

表 22 组治疗前后 GMFM-88 评分比较 $(\bar{x} \pm s)$

Table 2 Comparison of GMFM-88 scores between two groups before and after treatment $(\bar{x} \pm s)$

\begin{tabular}{|c|c|c|c|c|c|}
\hline \multirow{2}{*}{ 组 别 } & \multirow{2}{*}{$n$} & \multicolumn{2}{|c|}{ D 区评分 } & \multicolumn{2}{|c|}{$\mathrm{E}$ 区评分 } \\
\hline & & 治疗前 & 治疗后 & 治疗前 & 治疗后 \\
\hline 对照组 & 35 & $21.60 \pm 6.48$ & $24.89 \pm 6.25^{1)}$ & $26.37 \pm 10.17$ & $31.43 \pm 9.98^{13}$ \\
\hline 观察组 & 35 & $21.09 \pm 6.17$ & $28.37 \pm 5.84^{1 / 2)}$ & $26.83 \pm 10.67$ & $38.37 \pm 10.96^{122)}$ \\
\hline
\end{tabular}

注: 与治疗前比较, 1) $P<0.05$; 与对照组比较, 2) $P<0.05$ 。

Note: Compared with before treatment, 1) $P<0.05$; Compared with the control group, 2) $P<0.05$.

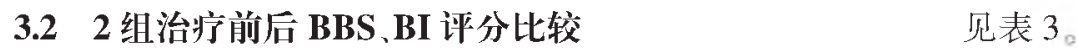

表 32 组治疗前后 BBS、BI 评分比较 $(\bar{x} \pm s)$

Table 3 Comparison of BBS, BI scores between two groups before and after treatment $(\bar{x} \pm s)$

\begin{tabular}{|c|c|c|c|c|c|}
\hline \multirow{2}{*}{ 组 别 } & \multirow{2}{*}{$n$} & \multicolumn{2}{|c|}{ BBS 评分 } & \multicolumn{2}{|c|}{ BI 评分 } \\
\hline & & 治疗前 & 治疗后 & 治疗前 & 治疗后 \\
\hline 对照组 & 35 & $25.11 \pm 7.14$ & $29.40 \pm 7.24^{1)}$ & $47.00 \pm 13.83$ & $55.71 \pm 14.04^{1)}$ \\
\hline 观察组 & 35 & $26.91 \pm 7.58$ & $38.63 \pm 7.25^{1 / 27}$ & $48.14 \pm 11.94$ & $66.86 \pm 12.43^{1 / 2)}$ \\
\hline
\end{tabular}

注: 与治疗前比较, 1) $P<0.05$; 与对照组比较, 2) $P<0.05$ 。

Note: Compared with before treatment, 1) $P<0.05$; Compared with the control group, 2) $P<0.05$.

\section{4 讨 论}

4.1 呼吸训练联合核心稳定性训练可提高 SCP 儿 童粗大运动功能

核心稳定性训练通过募集核心区域肌群,激活 更深层的稳定肌群, 协调稳定脊柱和骨盆, 使整体力
量的产生、传递和控制达到最佳化 ${ }^{[16]}$ 。本研究结果显 示, 2 组 GMFM-88 的 D 和 E 功能区评分较治疗前 均明显提高, 这提示核心稳定性训练可促进儿童粗 大运动功能的发育, 这与王永峰等 ${ }^{[7,11]}$ 研究结果一 致。观察组 GMFM-88 D 和 E 功能区评分明显高于 对照组, 这提示呼吸训练联合核心稳定性训练促进 
儿童粗大运动功能的发育效果更为显著。这与 $\mathrm{SON}$ 等 ${ }^{[17]}$ 研究发现核心稳定性训练配合呼吸训练可激 活 SCP 儿童重要呼吸肌、膈肌等深层核心肌肉,增强 核心稳定性并提高粗大运动功能和平衡能力的结果 一致。其作用机制可能与以下因素有关:(1)呼吸训 练可调节核心稳定性: 呼吸训练过程中重点关注 $\mathrm{SCP}$ 儿童胸廊护张、旋转的范围和质量以及胁间肌 的灵活性,使之在呼吸过程中更有效地工作, 通过 呼吸训练调节SCP 儿童的核心稳定性 ${ }^{[16]}$, 进而增强其 呼吸支持和呼吸协调性 ${ }^{[12]}$ 。(2) 呼吸训练可激活呼吸 肌群: 呼吸训练可激活膈肌和其他吸气肌, 增加呼 吸肌群肌力, 改善 SCP 儿童的肺功能, 同时增加腹内

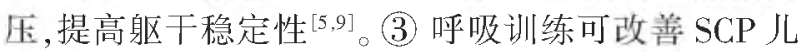
童呼吸模式: 呼吸训练可使 SCP 儿童浅而快的呼吸 模式逐步向深而缓的呼吸模式转变, 使吸气肌与呼 气肌运动更加协调, 儿童呼吸功能改善, 核心稳定 性增强, 运动功能的发育加快 ${ }^{[18]}$ 。

\section{2 呼吸训练联合核心稳定性训练可提高 SCP 儿} 童平衡能力

$\mathrm{SCP}$ 儿童常出现不同程度的平衡功能障碍, 引 发姿势及步态异常, 活动时易跌倒损伤, 进而影响患 儿的运动功能及日常生活能力 ${ }^{[19]}$ 。本研究结果显示, 2 组治疗后 BBS 评分较治疗前均明显提高, 这提示 核心稳定性训练可改善 SCP 儿童的平衡功能, 这与 李丹等 ${ }^{[8]}$ 研究结果一致。观察组治疗后 BBS 评分明 显高于对照组, 这提示呼吸训练联合核心稳定性训 练改善 SCP 儿童平衡能力的效果更为显著。其原因 可能与以下因素有关: (1) 核心稳定性训练可激活 核心肌群: 大部分胸腹部核心肌群 (如膈肌) 也是主 要的呼吸肌 ${ }^{[20]}$, 有维持姿势稳定和呼吸作用。核心稳 定性训练可使膈肌激活,再配合其他吸气肌训练可 有效提高 SCP 儿童的动态平衡能力 ${ }^{[9]}$ 。(2) 呼吸训练 可提高脊柱稳定性: 呼吸训练项目中的扩胸运动是 将深呼吸与躯干、四肢的主动运动结合起来的一种 全身运动技术, 有助于打开紧张的胸腔, 降低呼吸频 率, 扩大肋间隙, 增大胸腔容量, 提高吸气量和腹 压, 使SCP 儿童脊柱稳定性得到提高, 从而提高平衡 能力。(3)呼吸训练可改善运动模式: 呼吸训练可增 加肺活量, 更好地满足 SCP 儿童运动时血氧供应, 肌肉耐力增强, 运动时间更长, 运动模式逐渐正常化, 身体协调能力增强, 从而提高平衡能力。

\section{3 呼吸训练联合核心稳定训练可提高 SCP 儿童 日常生活活动能力}

日常生活活动与躯干稳定性和平衡能力密切相 关。本研究结果显示, 2 组治疗后 BI 评分较治疗前均
明显提高, 这提示核心稳定性训练可提高 SCP 儿童 的日常生活活动能力。观察组治疗后 BI 评分较对照 组明显更高, 这提示呼吸训练联合核心稳定性训练 提高 SCP 几童日常生活能力更为显著。这与 KELES 等 ${ }^{[9]}$ 研究发现膈肌等重要吸气肌的训练, 可增加吸气 肌的活动性及力量, 调节腹压, 增强 SCP 儿童躯干核 心稳定性, 增加活动耐力, 从而提高 SCP 儿童的日常 生活能力的结果一致。此外, SCP 儿童日常生活活动 能力与运动功能密切相关。有研究发现, 具有独立行 走能力的儿童比不能行走的儿童具有更好的呼吸肌 力量和肺功能, 呼吸肌力量与脑性㿈疾儿童日常生 活能力呈正相关 ${ }^{[21-22]}$ 。这提示, SCP 几童运动功能水 平越低, 日常生活能力也越低, 加强运动功能锻炼是 提高 SCP 几童日常生活能力的重要手段。因此, 本研 究通过呼吸训练配合核心稳定性训练可以有效改善 $\mathrm{SCP}$ 儿童粗大运动功能和平衡能力, 从而提高其日常 生活活动能力。

\section{5 小 结}

呼吸训练联合核心稳定性训练可有效改善 SCP 儿童粗大运动功能, 提高其平衡能力和日常生活活 动能力, 值得临床推广。但本研究还存在研究时间较 短、未进行出院后随访等不足之处, 下一步研究中 将开展大样本随机临床试验, 加强出院随访工作, 为 临床治疗 SCP 儿童运动功能障碍提供可靠依据。

\section{参考文献}

[1] 中国康复医学会儿童康复专业委员会, 中国残疾人康复协会小 儿脑性㿈癌康复专业委员会, 《中国脑性㿈癌康复指南》编委 会. 中国脑性㿈瘦康复指南 $(2015)$ : 第一部分 $[\mathrm{J}]$. 中国康复医 学杂志, 2015,30(7):747-754.

[2] ROSE J,CAHILL-ROWLEY K, BUTLER E E. Artificial walking technologies to improve gait in cerebral palsy: multichannel neuromuscular stimulation [J]. Artif Organs , 2017,41 (11) :E233E239.

[3] 任丽, 李淑琴. 核心稳定性训练对痉挛型脑性㿈疾患儿精细运 动功能的影响 $[\mathrm{J}]$. 康复学报, 2018,28(5):21-24.

[4] ERSOZ M,SELCUK B, GUNDUZ R, et al. Decreased chest mobility in children with spastic cerebral palsy [J]. Turk J Pediatr, $2006,48(4): 344-350$.

[5] HA S Y,SUNG Y H. Effects of Vojta approach on diaphragm movement in children with spastic cerebral palsy $[\mathrm{J}]$. J Exerc Rehabil, 2018,14(6): 1005-1009.

[6]朱丽蓉,董正婑,姚黎清. 脑㿈患儿照顾者生存质量及影响因 素的研究进展 $[J]$. 中国实用神经疾病杂志, 2019,22(1):103106.

[7]王永峰, 李晓捷, 吕洋, 等. 核心稳定性训练对痉孪型脑㿈患儿 粗大运动功能及步行能力的影响 $[\mathbf{J}]$. 中国康复理论与实践, $2012,18(4): 350-353$. 
[8] 李丹,刘军军,刘亚琼, 等. 核心稳定性训练对脑性㿈疻患儿功 能恢复的效果 $[\mathrm{J}]$. 中国康复理论与实践, 2015,21(5):583-585.

[9] KELES M N,ELBASAN B, APAYDIN U, et al. Effects of inspiratory muscle training in children with cerebral palsy: a randomized controlled trial $[J]$. Braz J Phys Ther, 2018,22(6):493501.

[10] PALISANO R,ROSENBAUM P, WALTER S, et al. Development and reliability of a system to classify gross motor function in children with cerebral palsy [J]. Dev Med Child Neurol,2008, $39(4): 214-223$.

[11] 解清云, 侯梅, 赵建慧, 等. 核心稳定训练对痉孪型脑性㿈疾患 儿运动功能的影响 $[\mathrm{J}]$. 中国康复医学杂志, 2014,29(6):528532,556 .

[12]刘起山, 莫昊风, 陈小芳. 呼吸训练对脑㿈患儿吞咽障碍的干 预效果分析 $[J]$. 康复学报,2019,29(1):27-32.

[13] 张玉梅, 宋鲁平. 康复评定常用量表 $[M]$. 北京: 科学技术文献 出版社, 2018:33-54.

[14] 何路, 徐开寿, 邱唒红, 等. Berg 平衡量表对痉挛型脑㿈儿童平 衡功能评定的信度研究 $[\mathrm{J}]$. 中国康复, 2010,25(1):21-23.

$[15]$ 黄东锋. 临床康复医学 (上册) $[\mathrm{M}]$. 汕头: 汕头大学出版社, $2004: 60-61$.
[16] 韩春远, 王卫星, 成波锦, 等. 核心力量训练的基本问题: 核心 区与核心稳定性 $[\mathrm{J}]$. 天津体育学院学报, 2012,27(2): 117120,172 .

[17] SON M S,JUNG D H, YOU J S H, et al. Effects of dynamic neuromuscular stabilization on diaphragm movement, postural control,balance and gait performance in cerebral palsy $[\mathrm{J}]$. Neuro Rehabilitation, 2017,41(4):739-746.

[18] 姚先丽, 是志远, 李坤新, 等. 等速肌力训练联合抗阻呼吸训练 对脑卒中后患者运动功能及日常生活能力的影响 $[\mathrm{J}]$. 广东医 学, 2019,40(24):3383-3387.

[19] 彭静, 王小伟, 孙冬梅, 等. 核心稳定性训练的研究进展 $[J]$. 中 国康复理论与实践, 2014,20(7):629-633.

[20]牛永刚. 人体运动涟功能训练结构模型研究 [D]. 石家生: 河北 师范大学, 2016:59-61.

[21] 郭云龙, 李晓捷, 孙奇峰, 等. 核心稳定性训练对痉挛型脑性㿈 痊患儿精细运动功能及日常生活活动能力的影响 $[\mathrm{J}]$. 中国中 西医结合儿科学, 2014,6(5):434-436.

[22] KWON Y H, LEE H Y. Differences of respiratory function according to level of the gross motor function classification system in children with cerebral palsy [J]. J Phys Ther Sci, 2014, 26 (3):389-391.

\title{
Effect of Respiratory Training combined with Core Stability Training for Children with Spastic Cerebral Palsy
}

\author{
HU Binong ${ }^{1}$, ZHANG Kai ${ }^{1}$, QI Fang ${ }^{1}$, WANG Chunhua ${ }^{2}$, ZHOU Wenyin ${ }^{2}$, AI Kun ${ }^{1 *}$, ZHANG Hong ${ }^{1 *}$ \\ ${ }^{1}$ College of Acupuncture-moxibustion and Massage, Hunan University of Traditional Chinese Medicine, Changsha, Hunan 410208, \\ China; \\ ${ }^{2}$ Xiangya Fraternity Rehabilitation Hospital, Changsha, Hunan 410000, China \\ *Correspondence: ZHANG Hong, E-mail: zh5381271@sina.com; AI Kun, E-mail:55509095@qq.com
}

\begin{abstract}
Objective: To observe the effect of respiratory training combined with core stability training on motor function, balance function and daily life ability of children with spastic cerebral palsy. Methods: A total of 74 children with spastic cerebral palsy who were treated in the deparment of child rehabilitation of Xiangya fraternity rehabilitation hospital from March to December 2019 , which were divided into the control group and the observation group according to the random number table, with 37 cases in each group. In the course of treatment, two cases dropped in each group, and finally 35 cases were included in each group. The control group was given core stability training on the basis of routine rehabilitation training. The routine rehabilitation training mainly included stretching training, joint range of motion training and occupational therapy, 10 minutes each time. Core stability training mainly included bypass training, sitting up training, suspension system training and Bobath ball training, 40 minutes per time. On the basis of routine rehabilitation training in the control group, the core stability training time of the observation group reduced from 40 minutes to 20 minutes each time, and then received respiratory training, 20 minutes each time. Respiratory training mainly includes abdominal respiratory training ( 7 min per time), chest expanding exercise training ( 3 min per time), respiratory aid method training ( 4 min per time), and anti blocking respiratory training ( $6 \mathrm{~min}$ per time). All the above items were trained twice a day, five times a week, continuous treatment for three months. Before and after treatment for three months, D and E functional domains of gross motor function measure scale- 88 (GMFM-88) was used to assess the gross motor function such as standing, walking, running and jumping; the Berg balance scale (BBS) was used to assess the balance function; and the Barthel index (BI) was used to assess the daily life activity ability. Results: Before treatment, there were no significant difference in the D and E functional domains scores of GMFM- 88 , BBS scores and BI scores between the two groups, with no statistically significant differences $(P>0.05)$. Compared with before treatment, the $\mathrm{D}$ and $\mathrm{E}$ functional domains of scores GMFM-88, BBS scores, BI scores of the both groups after treatment for three months were significantly improved, with statistically significant differences $(P<0.05)$. Compared with the control group, the D and $\mathrm{E}$ functional domains of scores GMFM-88, BBS scores, BI scores in the observation group were significantly higher than those of the control group, with statistically significant differences $(P<0.05)$. Conclusion: Respiratory training combined with core stability training can significantly improve the gross motor function, balance function and daily life ability of children with spastic cerebral palsy, which is worthy of clinical promotion.
\end{abstract}

KEY WORDS spastic cerebral palsy; respiratory training; core stability training; motor function; balance ability; activities of daily living

DOI : $10.3724 /$ SP.J.1329.2020.06006 